\title{
Innovative Research on Knowledge Service of University Library in the Background of Big Data
}

\author{
Yan Hu \\ Nanyang Institute of Technology, Henan, Nanyang, 473000
}

Keywords: Big Data; University Library; Knowledge Service

\begin{abstract}
It can be said that big data is the product of the development of science and technology and knowledge economy. The analysis of big data can comprehensively reflect the usage habits, laws and periods of users' information, enabling the data analysts to take corresponding measures according to the analysis results. For the university library, under the background of big data, readers' reading habit and reading environment have undergone qualitative changes. Under the new situation and new environment, the university library should give full play to its own advantages of massive scientific and technological literature resources, Data analysis, utilization, development, in order to improve the openness of knowledge and information to further innovate its own knowledge and services to better adapt to the current information environment. Based on the analysis of the relevance between big data and university libraries, this article summarizes the problems existing in the knowledge services of university libraries in the context of big data and puts forward some specific solutions.
\end{abstract}

\section{The Relevance of Big Data and the Library}

Big data, also known as massive resources and vast amounts of data, means that the amount of data involved is huge and cannot be processed within the reasonable time by using the existing mainstream software tools to help information analysts acquire positive information. This shows that the application of new processing model to analyze big data helps information analysts to obtain more decision-making, insightful, massive and diverse information and information. Big data has four notable features. The data volume is huge, the data types are modal, the data is generated rapidly, and the data is real-time. The data is huge in value but low in density, and its value is sparse. In addition, big data is different from traditional data model and data relationship in the way that the traditional first model generates data again. Big data first has data and then determines the mode, and the amount of data is in dynamic change. Moreover, the object of big data processing is not only data information, but also a basic resource. This basic resource can be used to solve problems in other fields. Therefore, big data analysis is an advanced analysis technique and is an application of a huge data set.

The application of big data in the field of library is the inevitable result of the digital development of the library. The library not only has a large number of electronic resources and digital resources, but also the massive data such as cloud computing and mobile library so the big data era of the library is already here. For university libraries, the number of data shows an explosive growth. Data types and data structures are becoming more and more diversified, and the data development value is getting higher and higher. Therefore, the overall quality and professional competence of librarians are also increasing the bigger Librarians should have the ability to mine and sort out the knowledge contents required by users by using big data analysis. Therefore, the demand for information processing ability and knowledge management ability of librarians in the big data era is getting higher and higher.

\section{The Problems of Current University Library Data Resource Management}

Although the data resources of major university libraries in China have accumulated more successful experiences in terms of open sharing, the development and application of big data in the 
field of domestic libraries still have a big gap compared with developed countries. Specifically, mainly reflected in the following areas:

First of all, there is a lack of overall planning for the open sharing of big data in university libraries, and there is a phenomenon of rebuilding and managing them lightly. There is not enough emphasis on the opening and sharing of information platforms. Each university library's big data innovation information resources are independent from each other, and there is a serious phenomenon of repeated construction of resources. The macroscopic management of data resources and overall coordination are not satisfactory. Second, the open data sharing system of big data lacks the necessary guarantee, and the relevant laws and regulations are in the form of formalities. The open operation of big data and data cannot be clearly defined or the authoritativeness of policies and laws is inadequate, which lacks the necessary effectiveness, resulting in the inability to open and apply knowledge services overall planning and adjustment. Knowledge data duplication, scattered, closed the problem has always existed, cannot provide the necessary protection for the open sharing of knowledge services in university libraries. Finally, big data innovation is under-supplied. Knowledge service of university library is not only to meet the reader's information needs, but also to provide the market main body necessary technical innovation and technological development support. However, in reality, it is very difficult for university libraries to directly serve the readers outside the library. Both the knowledge service awareness and the knowledge service fail to meet the established requirements. Therefore, university libraries should use big data technology to open up shared data resources and provide users with more high-quality knowledge services.

\section{The Application of Big Data Technology in College Library Knowledge Service}

Compared with public libraries, the advantage of knowledge resources of university libraries is very prominent. Library should make full use of its own advantages to realize the data collection, fusion, analysis and collation. By using knowledge visualization services, users can improve the convenience of acquiring knowledge, discover the reader's interest in service analysis, and then follow the reader's information industry to understand the reader's interest and knowledge needs. Specifically, we can build a library knowledge service engine system through the following technologies: First, knowledge visualization service. The so-called knowledge visualization is the use of data analysis technology for library big data integration, sorting, the knowledge content through the organization, analysis of real-time, the image displayed to the user, the visual service can better deal with a variety of complex heterogeneous data, which not only Can realize the retrieval process, the visualization of the retrieval structure, and can realize the visualization of the database distribution, the time distribution and the co-author relationship so as to make the other information related to the knowledge transparent and beneficial for the user to acquire and utilize the knowledge. Second, knowledge transfer services. Knowledge transfer service is based on data management technology, and integrates, processes and excavates the knowledge value of data resources in big data library, and analyzes the relevant information to solve the user's problems from the massive data; formulates the transfer target and realizes the data knowledge to the user The transfer of library services to enhance the capacity of knowledge. Through resource discovery technology, libraries can strengthen cooperation with other departments in the upstream and downstream industry chain of libraries to unify data standards and formats and analyze large quantities, types, disordered and heterogeneous data in libraries to form A library-centric knowledge base and metadata joint index library, in fact, with the library and other businesses, data providers seamless convergence in order to improve the efficiency of knowledge services.

Data is the basis of library application of big data technology. Therefore, it is necessary to construct a database that is easy to acquire, organize and manage big data resources, as follows: First, access to data resources. Library big data not only includes its own collection data, but also includes user data, dynamic RFID data, sensor data, scientific research shared data and mobile internet data, etc. The library must intelligently identify, perceive, adapt and transmit the big data source Etc., through the corresponding technology to build a data collection platform to collect data resources related to user knowledge needs. Second, organize data resources. If big data does not 
have a huge data size and does not establish a good data association, data analysis, mining, you cannot give full play to the value of big data, so the application of correlation technology to integrate the data into a data network and for example, university library can divide the data into basic level, service level, academic level, research level and so on, in order to improve the pertinence and efficiency of data source analysis. Library big data resources include structured, semi-structured, unstructured and other types of data, Hadoop and other HDFS file system can be structured, semi-structured, unstructured data effectively manage the data from time to time Organize, timely remove expired data, maintain new data archiving and more.

Therefore, under the background of big data, it is necessary to establish and improve the university library management system and ensure the management of big data knowledge service from the management level. Library is not only an important content in the organizational structure of colleges and universities, but also a system arrangement for university teachers and students to acquire knowledge and enjoy the right of knowledge.

First of all, college administrators should fulfill the responsibility of library management, establish a sound university library management system, and actively respond to the problems that arise in the application of library big data technology. Through the system constraints, adjust and optimize the knowledge of university library innovation service. Big data focuses on organizational analysis but requires strong analytical software and well-functioning system hardware as the foundation. University libraries have very limited funds. Therefore, school leaders should continue to increase their capital investment in libraries to provide in-depth analysis of big data Scalability of infrastructure support to improve the efficiency and effectiveness of data services. In addition, librarians should also constantly improve their own data analysis capabilities, actively pay attention to, learn relevant knowledge, and can more efficiently complete the acquisition, organization, management and analysis of user information behavior data and form decision-making capabilities. Users provide knowledge services in the process of continuous improvement of their ability to use big data. Second, do a good job of data security management, and effectively protect the privacy of users. University library big data includes a large number of user personal information, such as browsing history, loan records, geographical location, etc., which are more or less touched on the user's privacy issues; and university research information data involves a wider range of protection information Security is more important, so the library should do a good job of data security management, establish a digital privacy infrastructure, and create a benign environment for information. In addition, the library must strictly abide by the secret security system, make private use of the user's private information, and establish a solid trust mechanism with the user, reasonably and legally dig more user information, and further expand the source of big data information.

The open platform for university library sharing is not a linear and single innovation process. It requires multiple units to participate in it. All departments cooperate with each other to form the complementary advantages of each unit's data resources so as to improve the effectiveness of big data applications. The university library possesses massive and multi-type big data resources, which contains many latest scientific research achievements and produces a large amount of innovative information resources. Therefore, it plays a very important role in the sharing of big data knowledge service. Therefore, the university library should strengthen its cooperation with the government, intelligence agencies and other units, promote the development of the shared affiliate platform with the government as its lead, and understand the market demand for knowledge services through other intelligence agencies and enterprises. The extent to which data is openly shared enables unlimited access to knowledge resources for big data and continues to expand the value-added space for big data.

\section{Conclusion}

In short, under the background of big data, the knowledge service of university libraries has just started. There is no unified awareness and standard for the mining and acquisition of big data in the library community. However, it is undeniable that the application of big data technology in libraries can be continuously innovated. Therefore, it is foreseeable that big data technology is the new 
direction for the development of university library in the future.

\section{References}

[1] LI Ying. Research on Library Knowledge Service and Management in Big Data Environment [J]. Journal of Agricultural University of Chinese Academy of Sciences, 2016,28 (5): 168-170.

[2] Deng Fengyi, Deng Hairong. "Big tail effect" of digital publishing in the era of big data [J]. Publishing Research, 2014 (10): 27-29.

[3] Li Hongmei. On the impact of the era of big data on the history of the study [J]. Northern Forum, 2016 (2): 77-79.

[4] Lu Mingxin, Liu Zhaoshi, Sun Tingting et al. Impact of congestion on real-time traffic safety based on big data [J]. Shandong Transportation Science and Technology, 2016 (2): 12-15.

[5] Explain the Ming and Qing Dynasties. Big data samples and the overall relationship between the government statistics perspective [J]. China Statistics, 2014 (12): 54-55. 